\title{
Metallofullerene Series: Free-Metal Ionization-Potential Control of the Production Yields
}

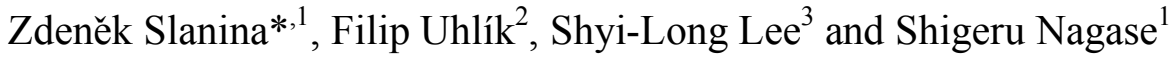 \\ ${ }^{I}$ Department of Theoretical and Computational Molecular Science, Institute for Molecular Science, Myodaiji, Okazaki \\ 444-8585, Aichi, Japan \\ ${ }^{2}$ Department of Physical and Macromolecular Chemistry, Charles University in Prague, Faculty of Science, Albertov 6 , \\ 12843 Prague 2, Czech Republic \\ ${ }^{3}$ Department of Chemistry and Biochemistry, National Chung-Cheng University, Chia-Yi 62117, Taiwan
}

\begin{abstract}
The paper reports computations for $\mathrm{Al} @ \mathrm{C}_{82}, \mathrm{Sc} @ \mathrm{C}_{82}, \mathrm{Y} @ \mathrm{C}_{82}$ and La@ $\mathrm{C}_{82}$ based on encapsulation into the IPR (isolated pentagon rule) $\mathrm{C}_{2 v} \mathrm{C}_{82}$ cage and also on $\mathrm{Mg} @ \mathrm{C}_{74}, \mathrm{Ca} @ \mathrm{C}_{74}, \mathrm{Sr} @ \mathrm{C}_{74}$ and $\mathrm{Ba} @ \mathrm{C}_{74}$ based on encapsulation into the only $\mathrm{C}_{74}$ IPR cage. Their structural and energetic characteristics are used for evaluations of the relative production yields, employing the encapsulation Gibbs-energy terms and saturated metal pressures. It is shown that the results can be well related to the ionization potentials of the free metal atoms.
\end{abstract}

Keywords: Endohedral fullerenes, carbon-based nanotechnology, molecular modeling, molecular electronic structure, ionization potentials, metallofullerene stabilities.

\section{INTRODUCTION}

There are several well-established families of metallofullerenes based on one common carbon cage, for example $\mathrm{X} @ \mathrm{C}_{74}$ or $\mathrm{Z} @ \mathrm{C}_{82}$. Although the empty $\mathrm{C}_{74}$ fullerene [1] is not yet available in solid form, several related endohedral species X@C $\mathrm{C}_{74}$ have been known like Ca@ $\mathrm{C}_{74}[2,3], \mathrm{Sr} @ \mathrm{C}_{74}$ [4], Ba@ $\mathrm{C}_{74}$ [5] or La@ $\mathrm{C}_{74}$ [6-8], all based on the isolated pentagon rule (IPR) $\mathrm{D}_{3 h} \mathrm{C}_{74}$ cage. Another common metallofullerene family, Z@ $\mathrm{C}_{82}$, is based on the IPR $C_{2 v} \mathrm{C}_{82}$ cage for example Sc@ $\mathrm{C}_{82}$ [9], Y@ $\mathrm{C}_{82}$ [10] and La@ $\mathrm{C}_{82}[6,11]$ (while $\mathrm{Al} @ \mathrm{C}_{82}$ was never isolated). The present paper deals with computational evaluations of the structural, bonding and stability features in the homologous series $\mathrm{Z} @ \mathrm{C}_{82}(\mathrm{Z}=$ Al, Sc, Y, La), and also X@ $\mathrm{C}_{74}(\mathrm{X}=\mathrm{Mg}, \mathrm{Ca}, \mathrm{Sr}, \mathrm{Ba})$. Special interest is paid to the Gibbs-energy evalutions for estimations of the relative populations.

Fullerenes and metallofullerenes have represented objects of very vigorous research activities in connection with their expected promising nanoscience and nanotechnology applications, see e.g. [12-17]. In particular, various endohedral cage compounds have been suggested as possible candidate species for molecular memories and other future molecularelectronic devices. One approach is built on endohedral species with two possible location sites of the encapsulated atom [13] while another concept of quantum computing aims at a usage of spin states of $\mathrm{N} @ \mathrm{C}_{60}$ [14] or fullerene-based molecular transistors [15]. Although there can be threedimensional rotational motions of encapsulates in the cages, the internal motions can be restricted by a cage derivatization

*Address correspondence to this author at the Department of Theoretical and Computational Molecular Science, Institute for Molecular Science, Myodaiji, Okazaki 444-8585, Aichi, Japan; Tel: 81-564-53-3258-3922;

E-mail: zdenek@ims.ac.jp
[16] thus in principle allowing for a versatile control of the endohedral positions needed for the molecular-memory applications. However, a still deeper knowledge of various molecular aspects of the endohedral compounds is needed before their tailoring to nanotechnology applications is possible.

\section{COMPUTATIONS}

The full geometry optimizations were carried out using density-functional theory (DFT), namely employing Beck's three parameter functional with the non-local Lee-Yang-Parr correlation functional (B3LYP) in the combined basis set of the 3-21G basis for $\mathrm{C}$ atoms and the LanL2DZ basis set with the LANL2 effective core potential for the metal atoms (3$21 \mathrm{G} \sim 1 \mathrm{a})$ as implemented in the Gaussian 03 program package [18]. In the optimized B3LYP/3-21G 1a geometries, the harmonic vibrational analysis was then performed. Moreover, in the optimized geometries, higher-level single-point energy calculations were also carried out with the standard 6$31+\mathrm{G}^{*}\left(6-31+\mathrm{G}^{*} \sim \mathrm{la}\right)$ basis set for $\mathrm{C}$ atoms, and finally also with the standard $6-311+\mathrm{G}^{*}$ basis for carbon atoms and the SDD basis with the SDD effective core potential for the metals $\left(6-311+\mathrm{G}^{*} \sim\right.$ sdd $)$. The B3LYP/3-21G la geometries have been known [12] to be comparable with the B3LYP/6$31 \mathrm{G}^{*}$ la structures for fullerenes and metallofullerenes. Moreover, at the B3LYP/3-21G la level the vibrational analysis is relatively feasible. The basis set superposition error (BSSE) was estimated by the Boys-Bernardi counterpoise method [19]. In addition to the traditional B3LYP functional, a newer MPWB1K functional suggested recently by Zhao and Truhlar [20] as the best combination for evaluations of long-range interactions has also partly been employed in this study. 
The Gibbs energies were evaluated using the rotationalvibrational partition functions constructed from the calculated structural and vibrational data using the rigid rotator and harmonic oscillator (RRHO) approximation, also applied in our previous study [21]. Although the temperature region where fullerene or metallofullerne electric-arc synthesis takes place is not yet known, there are some arguments to expect it around or above $1500 \mathrm{~K}$. Thus, the calculations here are presented for two illustrative temperatures of 1500 and $2000 \mathrm{~K}$.

\section{RESULTS AND DISCUSSION}

The Z@ $\mathrm{C}_{82}$ metallofullerenes have been known [12] to be formed via metal encapsulations into the IPR $C_{2 v} \mathrm{C}_{82}$ cage with a strong charge transfer from the metal to the cage leaving the metal between the $\mathrm{Z}^{2+}$ and $\mathrm{Z}^{3+}$ states. For example, the Mulliken atomic charge in La@ $\mathrm{C}_{82}, \mathrm{Y} @ \mathrm{C}_{82}$, and $\mathrm{Sc} @ \mathrm{C}_{82}$ is at the B3LYP/3-21G la level calculated as 2.67, 2.38, and 2.44 , respectively. However, the natural population analysis, for example at the B3LYP/6-311+G* sdd level, produces for La@ $\mathrm{C}_{82}, \mathrm{Y} @ \mathrm{C}_{82}$, and $\mathrm{Sc} @ \mathrm{C}_{82}$ charges of 2.32, 2.05, and 1.77 , respectively.

We can consider an overall stoichiometry of a metallofullerene formation:

$X(g)+C_{n}(g)=X @ C_{n}(g)$

although it is not really relevant what kind of reactants is on the left side as they will in the end cancel out in our considerations. The encapsulation process is thermodynamically characterized by the standard changes of, for example, enthalpy $\Delta H_{X @ C_{n}}^{\circ}$ or the Gibbs energy $\Delta G_{X @ C_{n}}^{\circ}$. An illustration is given here on the reaction series Al@ $\mathrm{C}_{82}, \mathrm{Sc} @ \mathrm{C}_{82}, \mathrm{Y} @ \mathrm{C}_{82}$ and La@ $\mathrm{C}_{82}$ with the encapsulation potential-energy changes computed at the B3LYP/6-31+G* la level. The original Boys-Bernardi counterpoise method was suggested [19] for dimers with a fixed geometry. Although a BSSE-respecting geometry optimization would be possible [22], it is rather practical only for simpler systems. Still, in order to reflect the cage distortion, a steric-corrected BSSE treatment is also applied here (B3LYP/6-31+G* la \& steric) which includes the difference between the energy of the carbon-cage geometry simply taken from $\mathrm{Z} @ \mathrm{C}_{82}$ and the energy of the related fully-optimized empty IPR $C_{2 v} \mathrm{C}_{82}$ cage.

The equilibrium composition of the reaction mixture is controlled by the encapsulation equilibrium constants $K_{X @ C_{n}, p}$ :

$K_{X @ C_{n}, p}=\frac{p_{X @ C_{n}}}{p_{x} p_{c_{n}}}$,

expressed in the terms of partial pressures of the components. The encapsulation equilibrium constant is interrelated with the standard encapsulation Gibbs energy change $\Delta G_{X @ C_{n}}^{\circ}$ :

$\Delta G_{X @ C_{n}}^{\circ}=-R T \ln K_{X @ C_{n}, p}$.

Temperature dependency of the encapsulation equilibrium constant $K_{X @ C_{n}, p}$ is then described by the van't Hoff equation: $\frac{d \ln K_{X @ C_{n}, p}}{d T}=\frac{\Delta H_{X @ C_{n}}^{\circ}}{R T^{2}}$

where the $\Delta H_{X @ C_{n}}^{\circ}$ term is typically negative so that the encapsulation equilibrium constants decrease with increasing temperature.

Let us further suppose that the metal pressure $p_{\mathrm{X}}$ is actually close to the respective saturated pressure $p_{X \text {,sat }}$. While the saturated pressures $p_{X \text {,sat }}$ for various metals are known from observations $[23,24]$ (and belong to the essential input set of experimental information [23-25] still necessary for our computational treatment), the partial pressure of $C_{n}$ is less clear as it is obviously influenced by a larger set of processes (though, $p_{C_{n}}$ should exhibit a temperature maximum and then vanish). Therefore, we avoid the latter pressure $C_{n}$ in our considerations at this stage, and this step can actually be done in a rigorous form. In order to observe the relative populations in a metallofullerene series, one can think on an experiment where all the considered metals are simultaneously placed in the electric-arc chamber. This experiment would obviously ensure the same conditions for every member of the series. Moreover, the term $p_{C_{n}}$ in eq. (2) will be in this arrangement just common for all the members of the series and thus, it can be canceled out.

Hence, we can just consider the combined $p_{X, s a t} K_{X @ C_{n}, p}$ terms:

$p_{X @ C_{n}} \sim p_{X, s a t} K_{X @ C_{n}, p,}$

that actually control the relative partial pressures of various $\mathrm{X} @ \mathrm{C}_{n}$ encapsulates in the endohedral series (based on one common $\mathrm{C}_{n}$ fullerene). In this way we get a simpler, applicable scheme. As already mentioned, the computed equilibrium constants $K_{X @ C_{n}, p}$ themselves have to show a temperature decrease with respect to the van't Hoff equation (eq. 4) which however does not necessarily mean a yield decrease with increasing temperature. Actually, the considered $p_{X, s a t} K_{X @ C_{n}, p}$ product term can frequently (though not necessarily) be increasing with temperature. An optimal production temperature could be evaluated in a more complex model that also includes temperature development of the empty-fullerene partial pressure.

Hence, if we want to evalute production abundances in a series of metallofullerenes like A1@ $\mathrm{C}_{82}, \mathrm{Sc} @ \mathrm{C}_{82}, \mathrm{Y} @ \mathrm{C}_{82}$ and $\mathrm{La} @ \mathrm{C}_{82}$, just the product $p_{Z, s a t} K_{Z @ C_{82}, p}$ terms can straightforwardly be used - some representative examples are shown in Table 1. While for $\mathrm{Al} @ \mathrm{C}_{82}$ the $p_{Z, s a t} K_{Z @ C_{82}, p}$ factor or quotient increases with temperature, it is about constant for $\mathrm{Y} @ \mathrm{C}_{82}$ for the considered temperatures, and it decreases with temperature for $\mathrm{Sc} @ \mathrm{C}_{82}$ and (especially) for $\mathrm{La} @ \mathrm{C}_{82}$. The behavior result from competition between the decreasing encapsulation equilibrium constants and increasing saturated-metal pressures. As the encapsulation enthalpy $\Delta G_{X @ C_{n}}^{\circ}$ has the most negative value for $\mathrm{La} @ \mathrm{C}_{82}$, its encapsulation equilibrium constant 3 has to exhibit the fastest temperature decrease that already cannot be overcompensated by the temperature increase of the saturated metal pressure so that 
Table 1. The Products of the Calculated ${ }^{a}$ Encapsulation Equilibrium Constant $K_{Z @ C_{22}, P}$ with the Metal Saturated-Vapor Pressure ${ }^{b}$ $p_{Z, \text { sat }}$ for $\mathrm{Al} @ \mathrm{C}_{82}, \mathrm{Sc} @ \mathrm{C}_{82}, \mathrm{Y} @ \mathrm{C}_{82}$ and La@C $\mathrm{C}_{82}$ Evaluated at Two Temperatures T

\begin{tabular}{|c|c|c|c|c|}
\hline \multirow[t]{2}{*}{ Endohedral } & \multicolumn{2}{|c|}{$p z$, sat $K_{Z @ C_{82}, P}$} & \multicolumn{2}{|c|}{$\frac{p z, \text { sat } K_{Z @ C_{82}, P}}{p_{L a, s a t} K_{L a @ C_{82}, P}}$} \\
\hline & $T=1500 \mathrm{~K}$ & $T=2000 \mathrm{~K}$ & $T=1500 \mathrm{~K}$ & $T=2000 \mathrm{~K}$ \\
\hline $\mathrm{Al} @ \mathrm{C}_{82}$ & $1.09 \times 10^{-6}$ & $3.77 \times 10^{-5}$ & $6.03 \times 10^{-8}$ & $4.99 \times 10^{-6}$ \\
\hline $\mathrm{Sc} @ \mathrm{C}_{82}$ & 5.16 & 3.66 & 0.286 & 0.484 \\
\hline $\mathrm{Y} @ \mathrm{C}_{82}$ & 2.57 & 2.93 & 0.142 & 0.388 \\
\hline $\mathrm{La} @ \mathrm{C}_{82}$ & 18.1 & 7.56 & 1.0 & 1.0 \\
\hline
\end{tabular}

${ }^{a}$ Computed with the B3LYP/6-31+G* la \& steric//B3LYP/3-21G la energetics and the B3LYP/3-21G la entropy.

${ }^{b}$ Extracted from available observed data $[22,23]$.

the $p z$,sat $K_{Z @ C_{82}, P}$ term decreases relatively so fast with temperature in this case. In order to allow for cancellation of various factors introduced by the computational approximations involved, it is however better to deal with the relative term $\frac{p z, \text { sat }_{Z} @ C_{82}, P}{p_{L a, s a t} K_{L a @ C_{82}, P}}$. The computed production yield of the (never observed) $\mathrm{A} 1 @ \mathrm{C}_{82}$ species should be by six orders of magnitude smaller than that for Sc@ $\mathrm{C}_{82}$ or $\mathrm{Y} @ \mathrm{C}_{82}$, while the latter two should exhibit comparable populations, and La@ $\mathrm{C}_{82}$ should be the most abundant endohedral in the series. This stability picture qualitatively agrees with observed populations [12]. In principle, an endohedral with lower value of the encapsulation equilibrium constant can still be produced in larger yields if a convenient over-compensation by higher saturated metal pressure can take place.

Although the energy terms are likely still not precise enough, their errors could be comparable in the series and thus, they should cancel out approximately in the relative term $\frac{p z, \text { sat } K_{Z @ C_{82}, P}}{p_{L a, s a t} K_{L a @ C_{82}, P}}$. This should be the case of, for example, the higher corrections to the RRHO partition functions, including motions of the encapsulate. The motion of the endohedral atom is highly anharmonic, however, its description is yet possible only with simple potential functions. As long as we are interested in the relative production yields, the anharmonic effects should at least to some extent be canceled out in the relative quotient $\frac{p z, s a t K_{Z @ C_{82}, P}}{p_{L a, s a t} K_{L a @ C_{82}, P}}$.

The series of metallofullerene formations with one common cage $\mathrm{X} @ \mathrm{C}_{n}$ allows for yet another interesting stability conclusion. Three formal reaction steps can be considered for our illustrative series $\mathrm{Al} @ \mathrm{C}_{82}, \mathrm{Sc} @ \mathrm{C}_{82}, \mathrm{Y} @ \mathrm{C}_{82}$ and $\mathrm{La} @ \mathrm{C}_{82}$ : (i) double- (or triple-) ionization of the free metal, (ii) double (or triple-) charging of the empty cage, and (iii) placing the metal di- (or tri-) cation into the di- (or tri-) anionic cage. Let us stress that these three steps are purely formal (as allowed in thermodynamic considerations) and they are not suggested as important steps in the real (unknown) formation mechanism. It should moreover be pointed out that throughout this paper we deal only with the observed ionization potentials and only for isolated (free) metal atoms.
The (ii) energy is identical for all members of the series, and the (iii) terms should be similar as they are controlled by electrostatics. The bonding situation in $\mathrm{Al} @ \mathrm{C}_{82}, \mathrm{Sc} @ \mathrm{C}_{82}$, $\mathrm{Y} @ \mathrm{C}_{82}$ and $\mathrm{La} @ \mathrm{C}_{82}$ can be surveyed by the highest $\mathrm{C}-\mathrm{Z}$ Wiberg bond index. The very low values of the $\mathrm{C}-\mathrm{Z}$ Wiberg index (at the B3LYP/6-311+G* $\sim$ sdd level: $0.04 \sim 0.21$ ) in $\mathrm{Z} @ \mathrm{C}_{82}$ indicate that instead of a covalent bond, an ionic bond is formed between the metal and cage. Moreover, the feature that the stabilization of metallofullerenes is mostly electrostatic was already documented [26] using the topological concept of 'atoms in molecules' (AIM) [27, 28] which indeed shows that the metal-cage interactions form ionic (and not covalent) bonds. The Wiberg-index analysis can be considered as an additional support for the finding. Hence, the free-metal ionization potentials should actually represent a critical yield-controlling factor - the computed relative potential-energy changes upon encapsulation $\delta_{\text {rel }} \Delta E$ and the relative observed ionization potentials of the free atoms $\delta_{\text {rel }} I P$ should according to the above three-step analysis be correlated:

$\delta_{\text {rel }} \Delta E \sim \delta_{\text {rel }} I P$.

This interesting conclusion is documented in Figs. ( 1 and 2). In the two figures, both the observed [25] second and third ionization potentials (IP) for the $\mathrm{Z}$ atom of the $\mathrm{Z} @ \mathrm{C}_{82}$ series are used as the $\mathrm{B} 3 \mathrm{LYP} / 3-21 \mathrm{G} \sim 1 \mathrm{a}$ Mulliken atomic charge on the metal in $\mathrm{Sc} @ \mathrm{C}_{82}, \mathrm{Y} @ \mathrm{C}_{82}$ and $\mathrm{La} @ \mathrm{C}_{82}$ is computed between 2 and 3. Fig. (1) presents the correlation for the $\mathrm{B} 3 \mathrm{LYP} / 6-31+\mathrm{G}^{*} \sim$ la relative potential-energy changes upon encapsulation $\delta_{r e l} \Delta E$, Fig. (2) for the B3LYP/6$31+\mathrm{G}^{*} \sim$ la $\&$ steric energetics. Finally, Fig. (3) deals with the $\mathrm{X} @ \mathrm{C}_{74}$ series [29] $(\mathrm{X}=\mathrm{Mg}, \mathrm{Ca}, \mathrm{Sr}$, and $\mathrm{Ba})$ described at the MPWB1K/6-31G* la level. In this case, both the observed second and first ionization potentials are considered (though the second IP are more relevant to this case as the Mulliken charge on the metals is very close to 2). The scheme works even better in this case owing to larger uniformity of the charge on the metals. All the three figures support relationship (6). In fact, such a correlation should operate for any homologous reaction series of metal encapsulation, i.e., into any type of a common carbon nanostucture. Moreover, this type of reasoning should step by step explain the fullereneencapsulation stability islands known throughout the periodic system though the underlying calculations are quite demanding [30-35]. While in this paper we treat reaction 


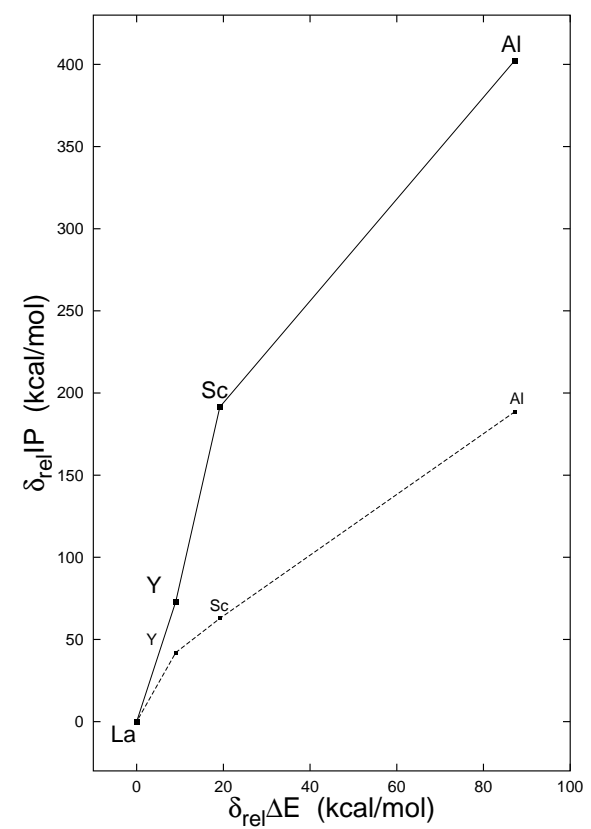

Fig. (1). The computed B3LYP/6-31+G* la relative potentialenergy changes upon encapsulation $\delta_{\text {rel }} \Delta E$ and the observed [24] relative ionization potentials (IP) of the free atoms $\delta_{\text {rel }} \mathrm{IP}$ for $\mathrm{Z} @ \mathrm{C}_{82}$ (solid line - 3-rd IP, dashed line - 2-nd IP).

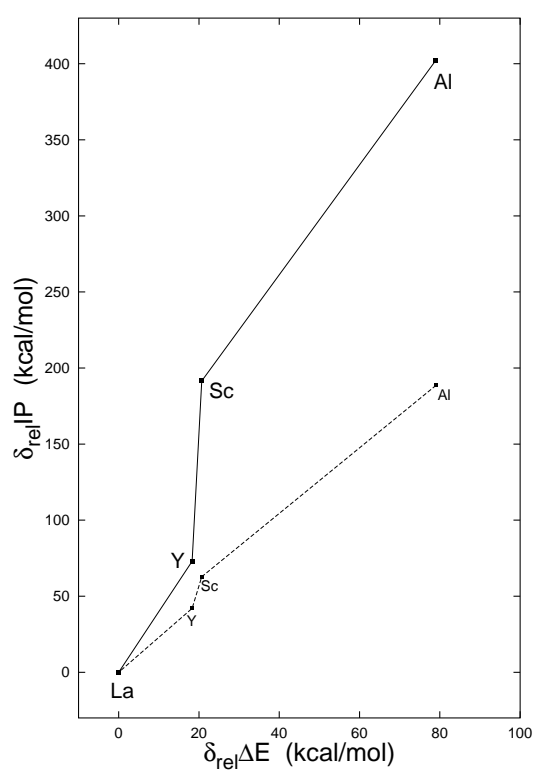

Fig. (2). The computed B3LYP/6-31+G* la \& steric relative potential-energy changes upon encapsulation $\delta_{r e l} \Delta \mathrm{E}$ and the observed [24] relative ionization potentials (IP) of the free atoms $\delta_{\text {rel }} I P$ for Z@ $\mathrm{C}_{82}$ (solid line-3-rd IP, dashed line-2-nd IP).

series along the columns of the periodic table, a similar treatment could be considered also along some of its rows.

In fact, we are dealing with a special case of clustering under saturation conditions [36-39]. The saturation regime is a useful simplification - it is well defined, however, it is not necessarily always achieved. Under some experimental arrangements, under-saturated or perhaps super-saturated metal vapors are also possible. This reservation is applicable not only to the electric-arc treatment but even more likely to newly introduced ion-bombardment production technique [40,41]. Still, eqs. (2) and (5) remain valid, however, the

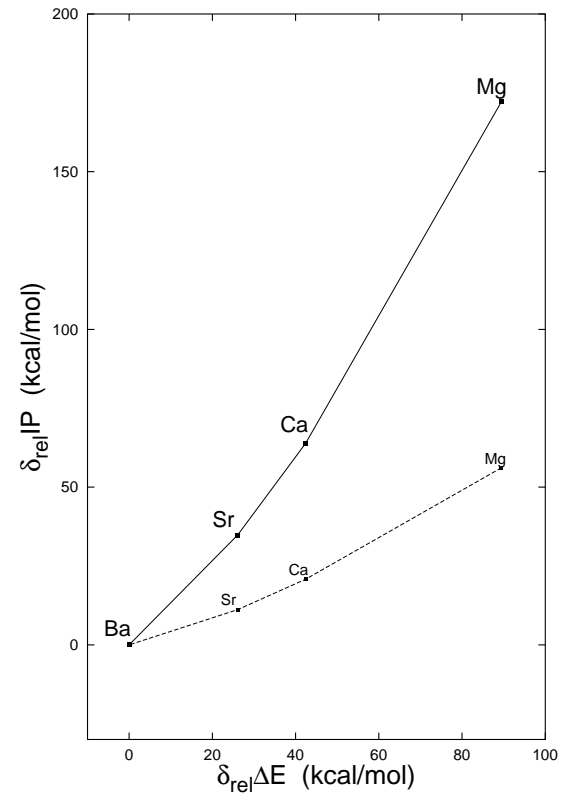

Fig. (3). The computed MPWB $1 \mathrm{~K} / 6-31 \mathrm{G}^{*} \sim$ la relative potentialenergy changes upon encapsulation $\delta_{r e l} \Delta E$ and the observed [24] relative ionization potentials (IP) of the free atoms $\delta_{\text {rel }} I P$ for the series $\mathrm{Mg} @ \mathrm{C}_{74}, \mathrm{Ca} @ \mathrm{C}_{74}, \mathrm{Sr} @ \mathrm{C}_{74}$, and $\mathrm{Ba} @ \mathrm{C}_{74}$ (solid line - the second IP, dashed line - the first IP).

metal pressure has to be described by the values actually relevant. For some volatile metals their critical temperature could be overcome and the saturation region thus abandoned (though practically speaking, this could come into consideration with mercury and cesium). Anyhow, the saturation regime can give a kind of upper-limit estimates of the production yields.

\section{ACKNOWLEDGMENTS}

The reported research has been supported by a Grant-inaid for the $21^{\text {st }}$ Century COE Program, Nanotechnology Support Project, the Next Generation Super Computing Project (Nanoscience Project), and Scientific Research on Priority Area from the Ministry of Education, Culture, Sports, Science, and Technology of Japan, by the National Science Council, Taiwan-ROC, and by the Ministry of Education of the Czech Republic (MSM0021620857) and the Czech Science Foundation/GACR (P208/10/0179). An initial phase of the research line was supported by the Alexander von Humboldt-Stiftung and the Max-Planck-Institut für Chemie (OttoHahn-Institut). Last but not least, referee's valuable comments are highly appreciated, too.

\section{REFERENCES}

[1] Diener MD, Alford JM. Isolation and properties of small-bandgap fullerenes. Nature 1998; 393: 668-71.

[2] Wan TSM, Zhang HW, Nakane T, et al. Production, isolation, and electronic properties of missing fullerenes: $\mathrm{Ca} @ \mathrm{C}_{72}$ and $\mathrm{Ca} @ \mathrm{C}_{74} . \mathrm{J}$ Am Chem Soc 1998; 120: 6806-7.

[3] Kodama T, Fujii R, Miyake Y, et al. C-13 NMR study of $\mathrm{Ca}_{0} \mathrm{C}_{74}$ : the cage structure and the site-hopping motion of a $\mathrm{Ca}$ atom inside the cage. Chem Phys Lett 2004; 399: 94-7.

[4] Haufe O, Hecht M, Grupp A, Mehring M, Jansen M. Isolation and spectroscopic characterization of new endohedral fullerenes in the size gap of $C_{74}$ to $C_{76}$. Z Anorg Allgem Chem 2005; 631: 126-30.

[5] Reich A, Panthofer M, Modrow H, Wedig U, Jansen M. The struc-

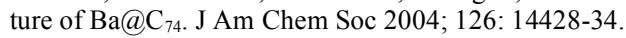


[6] Chai Y, Guo T, Jin C, et al. Fullerenes with metals inside. J Phys Chem 1991; 95: 7564-8.

[7] Sueki K, Akiyama K, Yamauchi T, et al. New lanthanoid metallofullerenes and their HPLC elution behavior. Full Sci Technol 1997; 5: $1435-48$.

[8] Nikawa H, Kikuchi T, Wakahara T, et al. Missing metallofullerene La@C 74 . J Am Chem Soc 2005; 127: 9684-5.

[9] Nishibori E, Takata M, Sakata M, Inakuma M, Shinohara H. Determination of the cage structure of $\mathrm{Sc} @ \mathrm{C}_{82}$ by synchrotron powder diffraction. Chem Phys Lett 1998; 298: 79-84.

[10] Takata M, Umeda B, Nishibori E, et al. Confirmation by X-ray diffraction of the endohedral nature of the metalfullerenes $\mathrm{Y} @ \mathrm{C}_{82}$. Nature 1995; 377: 46-8.

[11] Akasaka T, Wakahara T, Nagase S, et al. La@C82 anion. An unusually stable metallofullerene. J Am Chem Soc 2000; 122: 931617.

[12] Yamada M, Akasaka T, Nagase S. Endohedral metal atoms in pristine and functionalized fullerene cages. Acc Chem Res 2010; 43: 92-102.

[13] Gimzewski JK. Scanning tunneling and local probe studies of fullerenes. In: Andreoni W, Ed. The chemical physics of fullerenes 10 (and 5) years later. Dordrecht: Kluwer Academic Publishers 1996, pp. 117-36.

[14] Harneit W, Waiblinger M, Meyer C, Lips K. Weidinger A. Concept for quantum computing with $\mathrm{N} @ \mathrm{C}_{60}$. In: Kadish KM, Kamat PV, Guldi D, Eds. Recent Advances in the chemistry and physics of fullerenes and related materials, Fullerenes for the new millennium, Pennington: Electrochemical Society 2001, vol. 11: pp. 358-61.

[15] Hiroshiba N, Tanigaki A, Kumashiro R, Ohashi H, Wakahara T, Akasaka T. $\mathrm{C}_{60}$ field effect transistor with electrodes modified by La@C 82 . Chem Phys Lett 2004; 400: 235-8.

[16] Kobayashi K, Nagase S, Maeda Y, Wakahara T, Akasaka T. $\mathrm{La}_{2} @ \mathrm{C}_{80}$ : is the circular motion of two La atoms controllable by exohedral addition? Chem Phys Lett 2003; 374: 562-6.

[17] Gua G, Huang H, Yang S, et al. The third-order non-linear optical response of the endohedral metallofullerene Dy@ $\mathrm{C}_{82}$. Chem Phys Lett 1998; 289: 167-73.

[18] Frisch MJ, Trucks GW, Schlegel HB, et al. Gaussian 03, Revision C.01, Wallingford: CT Gaussian, Inc., 2004.

[19] Boys SF, Bernardi F. The calculation of small molecular interactions by the difference of separate total energies. Some procedures with reduced errors. Mol Phys 1970; 19: 553-66.

[20] Zhao Y, Truhlar DG. Benchmark databases for nonbonded interactions and their use to test density functional theory. J Chem Theor Comput 2005; 1: 415-32.

[21] Slanina Z, Uhlik F, Nagase S. Computed encapsulation energetics for metallofullerenes. Open Chem Phys J 2008; 1: 94-9.

[22] Simon S, Bertran J, Sodupe M. Effect of counterpoise correction on the geometries and vibrational frequencies of hydrogen bonded systems. J Phys Chem A 2001; 105: 4359-64.
[23] Alcock CB, Itkin VP, Horrigan MK. Vapor pressure equations for the metallic elements: 298-2500K. Can Metallurg Quart 1984; 23: 309-13.

[24] Lide DR, Ed. CRC Handbook of Chemistry and Physics. $85^{\text {th }}$ ed. Boca Raton, FL: CRC Press 2004, pp. 6-68-6-73.

[25] Lide DR, Ed. CRC Handbook of Chemistry and Physics, $85^{\text {th }}$ ed. Boca Raton, FL: CRC Press 2004, pp. 10-183-10-184.

[26] Kobayashi K, Nagase S. Bonding features in endohedral metallofullerenes. Topological analysis of the electron density distribution. Chem Phys Lett 1999; 302: 312-16.

[27] Bader RFW. A quantum theory of molecular structure and its applications. Chem Rev 1991; 91: 893-928.

[28] Bader RFW. A bond path: A universal indicator of bonded interactions. J Phys Chem A 1998; 102: 7314-23.

[29] Slanina Z, Uhlík F, Lee S.-L, Akasaka T, Nagase S. Carbon nanostructures: calculations of their energetics, thermodynamics and stability. In: Guldi DM, Martin N, Eds. Carbon nanotubes and related structures, Weinheim: Wiley-VCH Verlag 2010, pp. 491-523.

[30] Heine T, Vietze K, Seifert G. ${ }^{13} \mathrm{C}$ NMR fingerprint characterizes long time-scale structure of $\mathrm{Sc}_{3} \mathrm{~N} @ \mathrm{C}_{80}$ endohedral fullerene. Magn Reson Chem 2004; 42: S199-S201.

[31] Gurin VS. Endofullerenes with small silver and cooper clusters. Int J Quant Chem 2005; 104: 249-55.

[32] Valenica R, Rodriguez-Fortea A, Clotet A, et al. Electronic structure and redox properties of metal nitride endohedral fullerenes $\mathrm{M}_{3} \mathrm{~N} @ \mathrm{C}_{2 n}(\mathrm{M}=\mathrm{Sc}, \mathrm{Y}, \mathrm{La}$, and $\mathrm{Gd} ; 2 n=80,84,88,92,96)$. Chem Eur J 2009; 15: 10997-11009.

[33] Popov AA. Metal-cage bonding, molecular structures and vibrational spectra of endohedral fullerenes: bridging experiment and theory. J Comput Theor Nanosci 2009; 6: 292-317.

[34] Popov AA, Dunsch L. Bonding in endohedral metallofullerenes as studied by quantum theory of atoms in molecules. Chem Eur J 2009; 15: 9707-29.

[35] Contreras-Torres FF, Basiuk VA, Basiuk EV. Interaction between $\mathrm{NO}_{2}$ and an elongated fullerene $\mathrm{C}_{60}$. J Comput Theor Nanosci 2010; 7: 408-13.

[36] Slanina Z. Clustering, saturated vapors, and the atmosphere. J Chin Chem Soc 2003; 50: 607-10.

[37] Slanina Z. Clusters in a saturated vapor: pressure-based temperature enhancement of the cluster fraction. Z Phys Chem 2003; 217 : 1119-25.

[38] Slanina Z. Temperature development of mono- and heteroclustering in saturated vapors. J Clust Sci 2004; 15: 3-11.

[39] Slanina Z, Uhlík F, Lee S-L, Nagase S. Computational modeling for the clustering degree in the saturated steam and the watercontaining complexes in the atmosphere. J Quant Spectr Radiat Transf 2006; 97: 415-23.

[40] Murphy TA, Pawlik TH, Weidinger A, Höhne M, Alcala R, Spaeth $\mathrm{J}-\mathrm{M}$. Observation of atomlike nitrogen in nitrogen-implanted solid $\mathrm{C}_{60}$. Phys Rev Lett 1996; 77: 1075-8.

[41] Campbell EEB. Fullerene Collision Reactions. Dordrecht: Kluwer Academic Publishers 2003. 